\title{
Can the delayed phase of quantitative contrast-enhanced mammography improve the diagnostic performance on breast masses?
}

\author{
Weimin $\mathrm{Xu}^{1 \#}$, Bowen Zheng ${ }^{1 \#} \wedge$, Weiguo $\mathrm{Chen}^{1}$, Chanjuan Wen ${ }^{1}$, Hui Zeng ${ }^{1}, Z_{i l o n g} \mathrm{He}^{1}$, Genggeng Qin ${ }^{1}$, \\ Yingjia $\mathbf{L i}^{2}$
}

${ }^{1}$ Department of Radiology, Nanfang Hospital, Southern Medical University, Guangzhou, China; ${ }^{2}$ Department of Ultrasonic Medicine, Nanfang Hospital, Southern Medical University, Guangzhou, China

Contributions: (I) Conception and design: W Xu, B Zheng; (II) Administrative support: W Chen, G Qin, Y Li; (III) Provision of study materials or patients: C Wen, H Zeng; (IV) Collection and assembly of data: Z He, H Zeng; (V) Data analysis and interpretation: B Zheng, W Xu; (VI) Manuscript writing: All authors; (VII) Final approval of manuscript: All authors.

"These authors contributed equally to this work.

Correspondence to: Yingiia Li. Department of Ultrasonic medicine, Nanfang Hospital, Southern Medical University, Guangzhou 510515, China. Email: lyjia@smu.edu.cn; Genggeng Qin. Department of Radiology, Nanfang Hospital, Southern Medical University, Guangzhou 510515, China. Email: zealotq@smu.edu.cn.

Background: Contrast-enhanced mammography (CEM) is an imaging tool for breast cancer detection. Most quantitative analyses of CEM involve two phases, and it is unknown whether an added delayed phase can improve its diagnostic performance compared to dynamic contrast-enhanced magnetic resonance imaging (DCE-MRI). This study aimed to evaluate whether the delayed phase improves the diagnostic performance of CEM in distinguishing malignant and benign masses.

Methods: This prospective study enrolled 111 women with 111 pathologically confirmed breast masses. CEM was performed after the injection of contrast agent between 2-3 minutes (T1, early phase), 4-5 minutes (T2, second phase), and 7-9 minutes (T3, delayed phase). The quantitative enhanced gray value of lesions (LGV) and the lesion to background grey value ratio (LBR) were measured within each phase's corresponding region of interest (ROI). Based on their changes, the kinetic enhancement pattern was assessed among the three phases, and the diagnostic performance was subsequently measured.

Results: The LGV and LBR of malignant masses were significantly greater than those of benign lesions. The diagnostic performance of LGV and LBR at the delayed phase was consistent with that of the second phase but poorer than that of the early phase. The sensitivity of $\mathrm{LGV}_{\mathrm{T} 1}+\mathrm{LGV}_{\mathrm{T} 2}+\mathrm{LGV}_{\mathrm{T} 3}$ was less than that of $\mathrm{LGV}_{\mathrm{T} 1}+\mathrm{LGV}_{\mathrm{T} 2}(86.5 \%$ vs. 95.1\%) with a similar area under the curve (AUC), specificity, positivepredictive value (PPV), negative-predictive value (NPV), and accuracy. The sensitivity of $\mathrm{LBR}_{\mathrm{T} 1}+\mathrm{LBR}_{\mathrm{T} 2}+$ $\mathrm{LBR}_{\mathrm{T} 3}$ increased by $19.6 \%$, and specificity decreased by $20.7 \%$ compared with $\mathrm{LBR}_{\mathrm{T} 1}+\mathrm{LBR}_{\mathrm{T} 2}$. The $\mathrm{LGV}_{\mathrm{T} 1}$ $+\mathrm{LGV}_{\mathrm{T} 2}+\mathrm{LGV}_{\mathrm{T} 3}+$ kinetic enhancement $(\mathrm{T} 1-\mathrm{T} 3)$ had the lowest sensitivity $(67.0 \%)$, but the highest specificity (75.8\%), and the sensitivity of $\mathrm{LBR}_{\mathrm{T} 1}+\mathrm{LBR}_{\mathrm{T} 2}+\mathrm{LBR}_{\mathrm{T} 3}+$ kinetic enhancement (T1-T3) was higher than that of $\mathrm{LBR}_{\mathrm{T} 1}+\mathrm{LBR}_{\mathrm{T} 2}+$ kinetic enhancement (T1-T2) (90.2\% vs. 63.4\%, respectively).

Conclusions: The addition of a delayed CEM phase for breast cancer diagnosis yielded limited performance improvement. The quantitative analysis combined with enhancement patterns between the two consecutive phases has great potential to distinguish between malignant and benign lesions.

^ ORCID: 0000-0001-8653-8060. 
Keywords: Image enhancement; mammography; delayed-phase contrast-enhanced mammography (delayed-phase CEM)

Submitted Sep 23, 2020. Accepted for publication Apr 09, 2021.

doi: $10.21037 /$ qims-20-1092

View this article at: http://dx.doi.org/10.21037/qims-20-1092

\section{Introduction}

Contrast-enhanced mammography (CEM) is a breast cancer detection technique that uses an iodinated contrast agent and could provide morphologic and physiologic information similar to dynamic contrast-enhanced magnetic resonance imaging (DCE-MRI). CEM acquires lowenergy, and high-energy images in the craniocaudal (CC) and mediolateral oblique (MLO) views based on dualenergy mammography. The low-energy images are similar to standard mammography without the presence of iodine acquired at a range of $26-32$ peak kilovoltage $(\mathrm{kVp})$, below the K-edge of iodine. The high-energy images are acquired at a range of $45-49 \mathrm{kVp}$, above the K-edge, and display the iodine uptake in the breast tissue (1-4). By suppressing the normal background tissue enhancement, recombined images display breast lesions characterized by hyperangiogenesis due to the increased iodine uptake.

Morphological analysis is a fundamental method of breast lesion interpretation. However, certain malignant, precancerous, and benign lesions may share similar morphological characteristics $(2,5)$. The combination of morphologic information from low-energy images and physiological (enhancement) information from recombined images provided by CEM has been shown to demonstrate better sensitivity and specificity compared to full-field digital mammography (FFDM) and ultrasound (US), and a similar performance compared to MRI (3,4,6-8). Moreover, the Breast Imaging Reporting and Data System (BI-RADS) MRI lexicon can be used to describe enhanced lesions in recombined images, such as background parenchymal enhancement (BPE), enhancement patterns, and kinetic and parametric analysis.

Like MRI, BPE in contrast-enhanced spectral mammography (CESM) may indicate breast cancer (9). Rudnicki et al. (1) measured BPE by placing a region of interest (ROI) within the area of the most homogeneous subcutaneous fatty tissue. By placing an ROI over the most homogeneous enhancement areas within the lesion on two phases, the researchers obtained the lesion's quantitative enhancement. Further, they gained the percentage signal difference between enhancing the lesion and the background (\% RS) that achieved $88.6 \%$ specificity in differentiating between malignant and benign lesions. Consequently, they demonstrated that the stronger the enhancement, the higher the possibility of malignancy, and that quantitative analysis of CEM could distinguish between invasive cancers and benign or in situ lesions. However, they selected a small area within the breast parenchyma and the lesion and did not consider the whole breast tissue.

Previous studies have reported that the enhancement value's quantitative analysis can distinguish between benign and malignant lesions with high specificity (1,10-12). Deng et al. (10) analyzed the quantitative measurements and kinetic enhancement of benign and malignant lesions using CESM between two phases. They found that malignancies had stronger enhancement and depressed relative enhancement patterns than benign lesions. With an optimal cut-off enhanced value of 220.94 according to the Youden index, they achieved a $75.5 \%$ sensitivity, $88.6 \%$ specificity, and $82.1 \%$ accuracy.

As mentioned above, most quantitative analyses of CEM are based on two phases, which is not enough for kinetic enhancement analysis to achieve comparable diagnostic efficiency with MRI. Calibrated by a slab of breast tissueequivalent plastic filled with varying iodine contrast medium concentrations, Jong et al. (13) represented the lesion enhancement by iodine concentration. They computed the lesion enhancement at six phases and found that the most common kinetic enhancement for benign lesions was persistent, while the washout pattern was most common for the malignancies. However, this study enrolled only 22 lesions, with 13 being enhanced. Huang et al. (14) explored the breast lesion kinetic patterns at 2, 3, 4, 7, and $10 \mathrm{~min}$ after the contrast agent injection. Classified as persistent, plateau, and washout, they found that benign and malignant lesions manifested the highest enhancement at 3 and $2 \mathrm{~min}$, respectively, and the washout pattern was associated with the malignancies at $2-4$ and $2-10$ min periods. However, 
Figure 1 Imaging acquisition order. The abnormal breasts were scanned three times in the following order: CC view, MLO view, and the second MLO view, which were performed at 2, 4, and 7 minutes, and completed within 3, 5, and 9 minutes after the injection of the contrast medium, respectively. CC, craniocaudal; MLO, mediolateral oblique.

Huang et al. obtained the enhancement by visual and semiquantitative analysis from three readers, resulting in a subjective bias.

By objectively analyzing the quantitative enhancement of breast lesions, this study aimed to explore whether the delayed phase improved the diagnostic performance of CEM in distinguishing malignant and benign lesions and attempted to facilitate the detection and characterization of breast lesions.

\section{Methods}

\section{Selection of study participants}

The study was conducted in accordance with the Declaration of Helsinki (as revised in 2013). The Ethics Committee of Nanfang Hospital, Southern Medical University approved this prospective study (NFEC-2017136), and written informed consent was obtained from all enrolled participants.

From January 2018 to October 2019, all-female patients who underwent CEM at our hospital were enrolled in the study. The inclusion criteria were as follows: (I) patients with suspicious masses in the breast on FFDM or US or both, and those who then underwent CEM on a Senographe Essential (GE Healthcare CESM) at our institute. (II) CEM that was performed in the following order: (i) CC view, lateral projection of the abnormal side, (ii) CC view, lateral projection of the normal side, (iii) MLO view, lateral projection of the abnormal side, (iv) MLO view, projection of the normal side, (v) MLO view, lateral projection of the abnormal side. (III) Breast lesions demonstrating enhancement on recombined images; (IV) breast lesions that were confirmed pathologically after incisional biopsy or after complete excision. The MLO view was chosen over the $\mathrm{CC}$ view for the delayed phase projection as it involves more breast tissue and displays the lesion better. The exclusion criteria were as follows: (I) patients with an estimated glomerular filtration rate (eGFR) less than $30 \mathrm{~mL} / \mathrm{min}$ according to previous blood biochemistry tests; (II) patients who were allergic to iodinated contrast agents; (III) patients with breast implants; and (IV) patients who had more than one enhanced lesion on recombined images.

\section{Imaging acquisition}

Before the imaging acquisition, a single bolus injection of non-ionic contrast medium (Omnipaque, Bayer, Whippany, $\mathrm{NJ}$, USA; $300 \mathrm{mgI} / \mathrm{mL}$ ) was administered via an intravenous catheter at an injection rate of $3 \mathrm{~mL} / \mathrm{s}$ and a dose of $1.5 \mathrm{~mL} / \mathrm{kg}$. After considerable compression, the low-energy (26-30 kVp) and high-energy (45-49 kVp) exposures were performed in CC and MLO views for each breast. The recombined images were obtained by diminishing the attenuation differences between the low-energy and highenergy images and reducing the non-enhancing image's noise. The abnormal breast was assessed first, followed by the normal breast. The average glandular radiation (AGD) of each view was calculated for further analysis.

The abnormal breasts were scanned three times in the following order (Figure 1): CC view, MLO view, and the second MLO view performed at 2, 4, and 7 minutes, and completed within 3, 5, and 9 minutes after the injection of the contrast medium. The early phase was defined as $T_{1}$ (2-3 min after the injection of the contrast medium in CC views); the second phase was defined as $\mathrm{T}_{2}(4-5 \mathrm{~min}$ after injection in MLO views); the delayed phase was defined as $\mathrm{T}_{3}$ (7-9 min after injection in MLO views).

\section{Imaging analysis}

All images were transformed into 16-bit gray-level images (range, 0-65,536). A radiologist with 8 years of experience in breast imaging manually contoured and adjusted the ROI of each mass on recombined images using a free-hand 

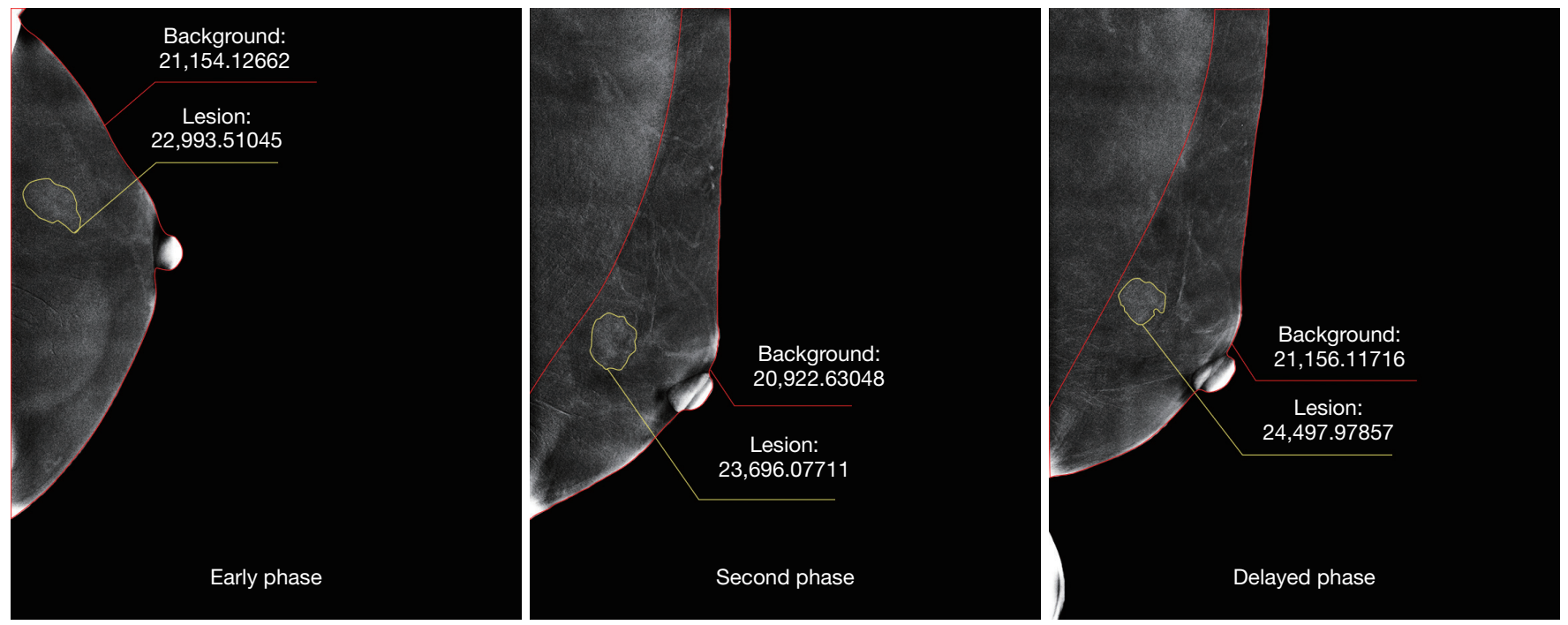

Figure 2 Measuring the enhancement of masses on a contrast-enhanced image achieved by weighted logarithmic subtraction. Lesion ROIs were manually contoured around the enhancing lesion edge while the background ROIs were obtained using the breast contour minus the lesion ROI and extreme high-density points such as the nipple and skin folds. The MLO view would go further minus the area of the pectoralis major. ROI, region of interest; MLO, mediolateral oblique.

dragging tool in ITK-SNAP (version 3.8.0; Cognitica, Philadelphia, PA, USA) along the edge of the lesion. Since we could not separately obtain the exact pixel values of the normal glandular tissue, which was superimposed over the enhanced lesions, the results of enhanced values were based on the bounded lesions (Figure 2). The background ROIs were obtained using the breast contour minus the lesion ROI and extreme high-density points, such as the nipple and skin folds, using a threshold enhanced value. The MLO view could reach other areas, minus the area of the pectoralis major. Using Simple ITK (version 1.2.0; Cognitica) and Open CV (version 4.1.2) in Python 3.7 (Python Software Foundation, https://www.python.org/) for matching the ROIs with corresponding recombined images, the BPE and quantitative enhanced gray value of lesions (LGV) were measured. The lesion to background grey value ratio (LBR) and the lesion enhanced value difference at different phases (LGVD) was further calculated utilizing the following equations \{Eqs. [1] and [2]\}:
According to the ACR-MRI recommendations (15), kinetic enhancement patterns based on the change in LGV and LBR between two adjacent phases were divided into three patterns as follows: (I) persistent, change $>10 \%$; (II) plateau, change of $-10 \%$ to $\leq 10 \%$; (III) washout, change $<-10 \%$ (Figure 3).

The lesions were classified based on the quantitative LGV and LBR values and the corresponding kinetic enhancement patterns between two consecutive phases and all three phases. The lesions classified as over BI-RADS 4B were judged to be malignant, and lesions over the BI-RADS 4A category would be pathologically confirmed by biopsy or by surgery.

\section{Statistical analysis}

All statistical analyses were performed using SPSS 22.0 statistical software (IBM Corp., Armonk, NY, USA). The diagnostic accuracy of CEM for discriminating between

$$
\begin{aligned}
& \text { Equation } 1: L B R=\frac{\text { Greyvalue }_{\text {lesion }}-\text { Greyvalue }_{\text {background }}}{\text { Greyvalue }_{\text {background }}} \\
& \text { Equation 2: } L G V D=\frac{\text { Lesion greyvalue }_{\text {latter phase }}-\text { Lesion grey value }_{\text {previous phase }}}{\text { Lesion greyvalue }_{\text {previous phase }}} \times 100 \%
\end{aligned}
$$




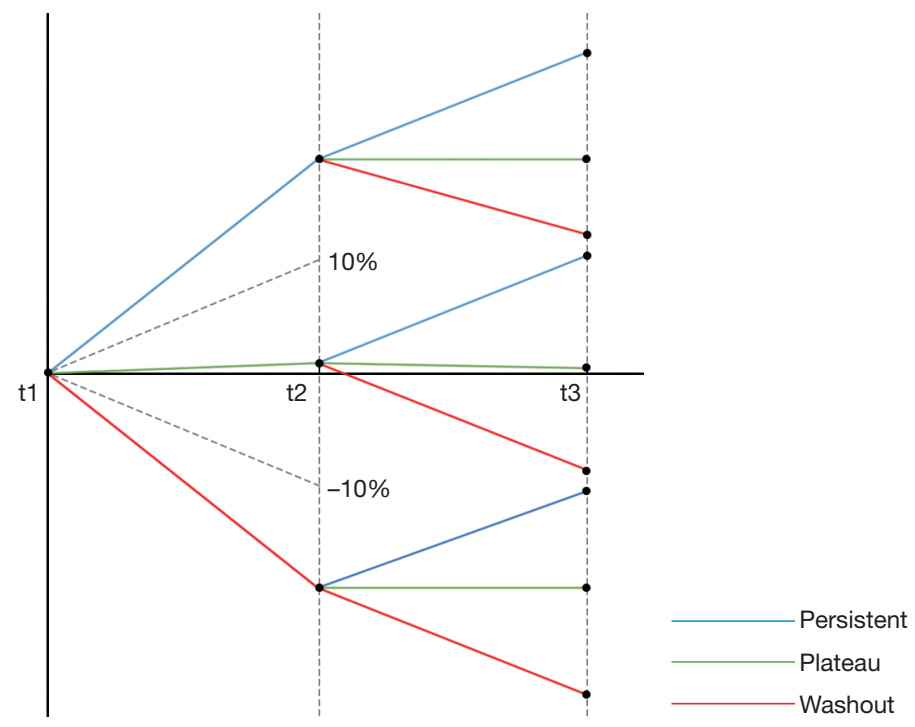

Figure 3 Enhancement patterns of the early, second, and delay phases. Persistent pattern: the interval enhancement increased more than $10 \%$. Plateau pattern: the interval enhancement changed within $10 \%$. Washout pattern: the interval enhancement decreased more than $10 \%$.

benign and malignant masses was analyzed statistically using Pearson's $\chi^{2}$ test and receiver operating characteristic (ROC) curve analysis. The best cut-off value was determined according to the Youden index, in which $\mathrm{Y}=$ (sensitivity + specificity) -1 . Regarding the roles of the quantitative variables of lesions in differentiating malignancy from a benign lesion, sensitivity, specificity, and accuracy were calculated by regarding the variables as the discrimination criteria and cut-off points. The differences between LGV, LBR, and BPE in benign and malignant lesions among different phases were analyzed using a one-way analysis of variance. The enhanced degree of lesions, the lesions based on the quantitative LGV and LBR values, and the corresponding kinetic enhancement patterns between two phases and three phases were analyzed statistically by a Pearson's $\chi^{2}$ test. A P value $<0.05$ was considered to indicate statistical significance.

\section{Results}

From January 2018 to October 2019, 124 female patients were enrolled based on the inclusion and exclusion criteria. Among them, eight patients were further excluded as they opted out due to poor economic conditions, and two patients were excluded because of blur and artifact in their diagnostic images. Further, three patients were excluded because of extreme BPE on their diagnostic images, resulting in 111 patients finally enrolled in the study.
All lesions were confirmed pathologically by incisional biopsy or after excisional surgery. The predominant breast composition categories were $\mathrm{c}$ and $\mathrm{d}$, with a combined 96/111 (86.4\%). Remarkably, all lesions (111 single masses on 111 laterals breasts) were enhanced on the recombined images. The average time interval between the CC and the second lateral MLO views of the abnormal breast was $263 \mathrm{~s}$, and the average time interval of the bilateral breast was $338 \mathrm{~s}$ (range 169 to $458 \mathrm{~s}$ ).

Patients were subjected to a mean average glandular dose (AGD) of 1.92 $\pm 0.05 \mathrm{mGy}$ (range, 1.06-3.64 mGy) per view for a breast thickness of $48.57 \pm 0.43 \mathrm{~mm}$ under compression. The mean AGD of the second MLO view was $1.98 \pm 0.06 \mathrm{mGy}$ (range, $1.05-3.76 \mathrm{mGy}$ ) per image for a breast thickness of $50.49 \pm 1.08 \mathrm{~mm}$. Because of the addition of the second MLO view, we calculated the total AGD of the five projections to be $9.60 \pm 0.25 \mathrm{mGy}$, ranging from 5.32 to 18.20 mGy per patient.

Eighty-two (73.8\%) breast masses were eventually confirmed as malignant on pathological examination comprising 72 invasive ductal carcinomas (IDCs), one ductal carcinoma in situ (DCIS), and 9 other subtypes. The remaining $29(26.2 \%)$ masses were benign on pathological examination (Table 1).

\section{Quantitative variables between benign and malignant lesions}

The mean LGV, LBR, and BPE were measured and 
Table 1 Patient clinical characteristics

\begin{tabular}{|c|c|c|}
\hline Characteristics & $\begin{array}{c}\text { Malignant } \\
\quad(n=82)\end{array}$ & $\begin{array}{l}\text { Benign } \\
(n=29)\end{array}$ \\
\hline \multicolumn{3}{|l|}{ Gender, n (\%) } \\
\hline Female & $82(100.0)$ & $29(100.0)$ \\
\hline Age, range (years) & $28-74$ & $24-65$ \\
\hline Median (years) & 48.42 & 45.24 \\
\hline \multicolumn{3}{|l|}{ BI-RADS breast density, n (\%) } \\
\hline a & $3(3.7)$ & $0(0.0)$ \\
\hline $\mathrm{b}$ & $7(8.5)$ & $5(17.2)$ \\
\hline c & $60(73.2)$ & $17(58.7)$ \\
\hline$d$ & $12(14.6)$ & $7(24.1)$ \\
\hline \multicolumn{3}{|l|}{ Pathology, n } \\
\hline \multicolumn{3}{|l|}{ Malignant } \\
\hline DCIS & 1 & \\
\hline IDC & 72 & \\
\hline Other types & 9 & \\
\hline \multicolumn{3}{|l|}{ Benign } \\
\hline Fibroadenoma & & 5 \\
\hline Intraductal papilloma & & 4 \\
\hline Inflammation & & 7 \\
\hline Phyllodes tumors & & 3 \\
\hline Adenosis & & 4 \\
\hline Fibrosis disease & & 3 \\
\hline Cysts combined inflammation & & 3 \\
\hline
\end{tabular}

BI-RADS, Breast Imaging Reporting and Data System; DCIS, ductal carcinoma in situ; IDC, invasive ductal carcinoma.

compared. The LGV and LBR of breast cancer were higher than those of benign lesions in the early and second phases, and the mean LGV and LBR decreased for malignant lesions and increased for benign lesions over time. The LGV and LBR were significantly different between malignant and benign lesions in the early phase $(\mathrm{P}<0.05)$ instead of the second or the delayed phase $(\mathrm{P}>0.05$, Table 2$)$. The difference in LGV in malignant and benign lesions reached the maximum in the early phase, with a minimum LGV of benign lesions of 27,206.8 and a maximum LGV of malignancies of $31,934.4$. Furthermore, the difference reached its minimum in the delayed phases, with benign lesions and malignancies having similar average LGVs (benign vs. malignant: 29,057.8 vs. 29,705.2). The LBR differences shared a similar pattern, with a maximum in the early phase (benign $v s$. malignant: $0.294 v s .0 .517$ ) and a minimum in the delayed phase (benign vs. malignant: 0.437 vs. 0.413), and the BPE was not significantly different in malignant and benign lesions in each phase $(\mathrm{P}>0.05$, Table 2$)$. The average $\mathrm{BPE}$ of the benign versus malignant lesions was $20,995.5$ vs. 21,032.1 for T1, 20,635.5 vs. 20,682.7 for $\mathrm{T} 2$, and 20,635.2 vs. 20,659.5 for T3.

\section{The diagnostic performance of $L G V$ and $L B R$ among different imaging phases based on ROC}

Table 3 shows the diagnostic performance of LGV and LBR among different phases. For each of these, we ascertained that LGV and LBR could discriminate malignant from benign lesions based on ROC analysis [area under the curve (AUC) $>0.5$; Table 3, Figure 4].

Based on the LGV, the AUC, sensitivity, positivepredictive value (PPV), negative-predictive value (NPV), and accuracy in the early phase were higher than those in the second and delayed phase, with $93.9 \%$ sensitivity and 81.9\% accuracy. Based on LBR, the AUC, sensitivity, and accuracy in the early phase were higher than those in the second and delayed phases, with an AUC of $0.708,86.5 \%$ sensitivity, and $77.4 \%$ accuracy (Table 3 ).

We further explored the diagnostic performance between two consecutive phases and three phases (Table 4). Compared with $\mathrm{LGV}_{\mathrm{T} 1}+\mathrm{LGV}_{\mathrm{T} 2}$, the sensitivity of $\mathrm{LGV}_{\mathrm{T} 1}$ $+\mathrm{LGV}_{\mathrm{T} 2}+\mathrm{LGV}_{\mathrm{T} 3}$ was lower (86.5\% vs. $95.1 \%$ ), while the AUC, specificity, PPV, NPV, and accuracy were similar. For $\mathrm{LBR}_{\mathrm{T} 1}+\mathrm{LBR}_{\mathrm{T} 2}+\mathrm{LBR}_{\mathrm{T} 3}$, the sensitivity was higher than that of $\mathrm{LBR}_{\mathrm{T} 1}+\mathrm{LBR}_{\mathrm{T} 2}(93.9 \%$ vs. $74.3 \%)$, while the specificity was lower than that of $\mathrm{LBR}_{\mathrm{T} 1}+\mathrm{LBR}_{\mathrm{T} 2}(37.9 \%$ vs. $58.6 \%)$.

\section{Comparison of the enhancement value parameters with the qualitative evaluation by the reader}

The qualitative assessment of enhancement and the quantitative assessment of LGV and LBR are shown in Figures 5,6, demonstrating equivalent conspicuity and confidence levels on qualitative evaluation by the reader and quantitative assessment of LGV and LBR. Therefore, the combination of qualitative assessment and kinetic enhancement patterns showed similar performance compared to LGV and LBR combined with kinetic enhancement patterns. 
Table 2 Comparison of LGV, LBR, and BPE values between malignant and benign lesions in different phases

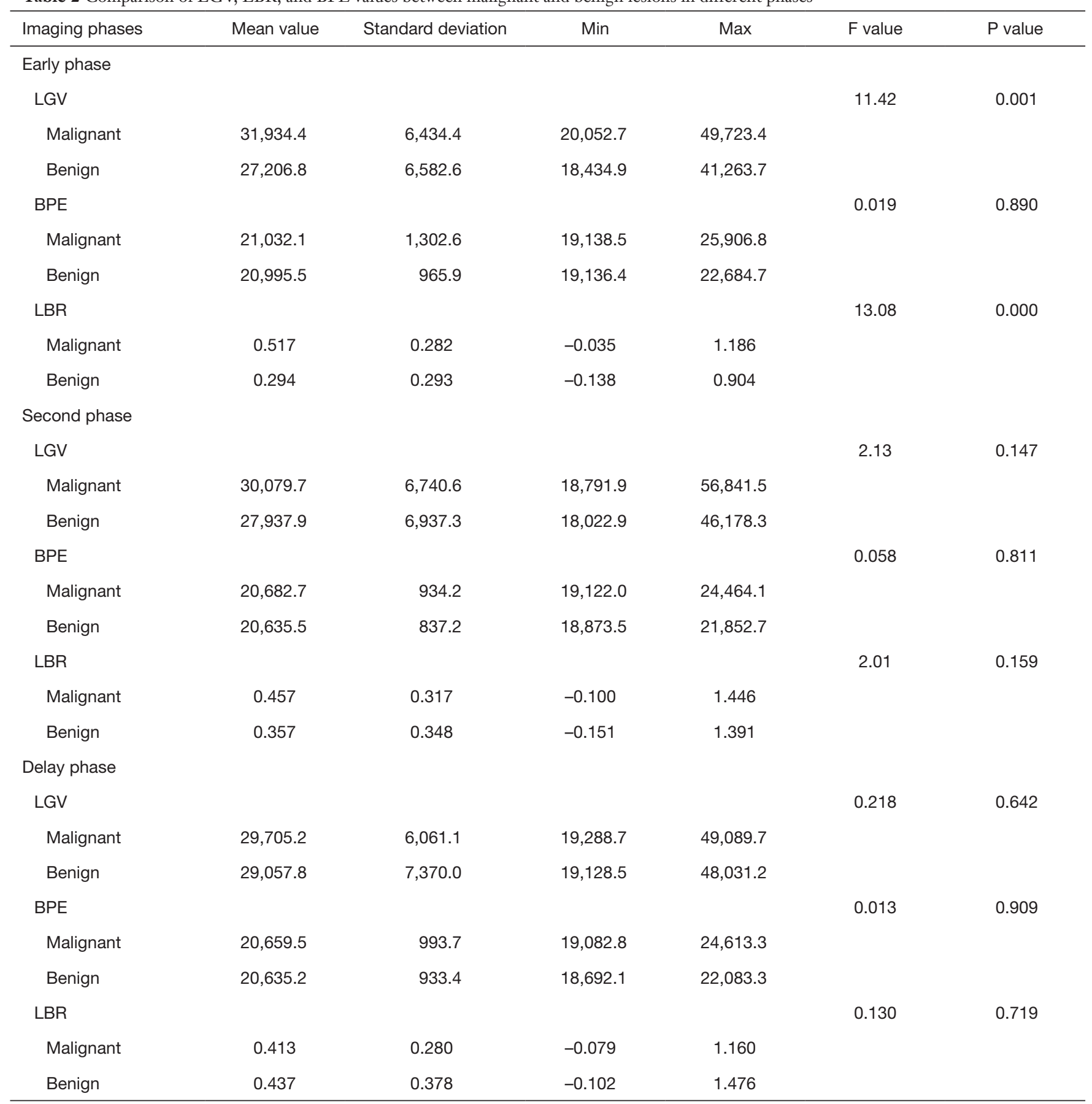

LGV, quantitative enhanced gray value of lesions; LBR, lesion to background grey value ratio; BPE, background parenchymal enhancement.

The diagnostic performance based on the $L G V$ and $L B R$ combined with kinetic enhancement patterns between malignant and benign lesions

We further explored the kinetic enhancement patterns of
LGV of two consecutive phases and of three consecutive phases (Figure 7) and found the kinetic enhancement patterns of benign and malignant lesions were significantly different between the two consecutive phases $(\mathrm{P}<0.05)$. 
Table 3 Comparing the diagnostic performance of LGV and LBR alone among different imaging phases

\begin{tabular}{|c|c|c|c|}
\hline Diagnostic performance & \multicolumn{3}{|c|}{ Imaging phases } \\
\hline \multicolumn{4}{|l|}{ LGV } \\
\hline AUC & 0.711 & 0.605 & 0.557 \\
\hline Sensitivity (\%) & 93.9 & 76.8 & 87.8 \\
\hline PPV (\%) & 83.6 & 81.8 & 78.2 \\
\hline NPV (\%) & 73.6 & 44.1 & 47.3 \\
\hline Accuracy (\%) & 81.9 & 70.2 & 72.9 \\
\hline \multicolumn{4}{|l|}{ LBR } \\
\hline Specificity (\%) & 51.7 & 44.8 & 51.7 \\
\hline PPV (\%) & 83.5 & 80.4 & 80.5 \\
\hline NPV (\%) & 57.6 & 44.8 & 38.4 \\
\hline Accuracy (\%) & 77.4 & 71.1 & 65.7 \\
\hline
\end{tabular}

LGV, quantitative enhanced gray value of lesions; LBR, lesion to background grey value ratio; AUC, area under the curve; PPV, positivepredictive value; NPV, negative-predictive value.

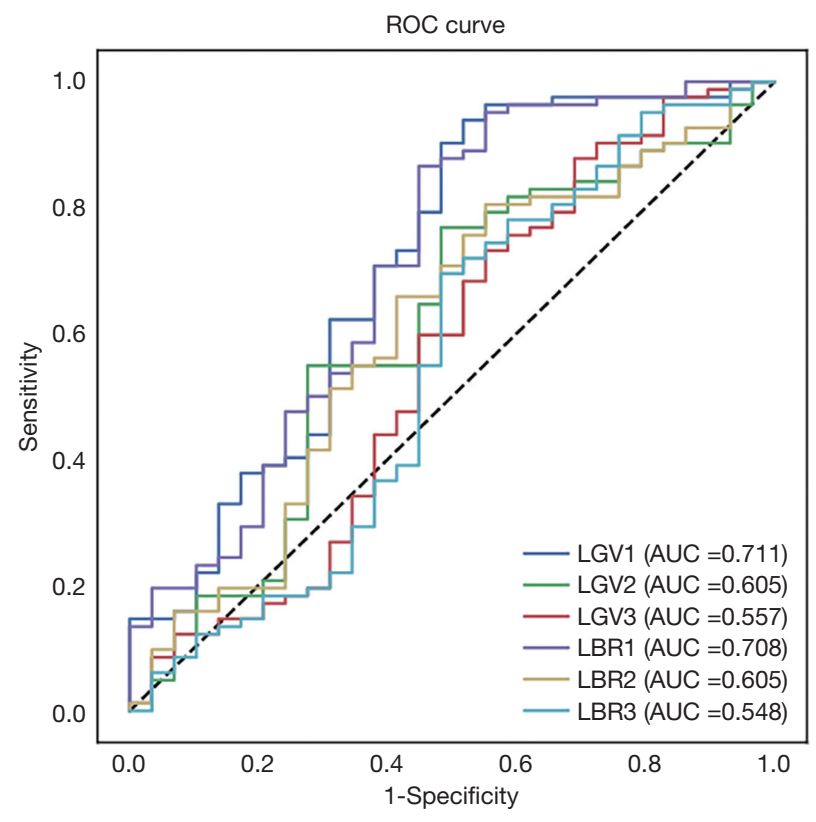

Figure 4 The diagnostic value of the LGV and LBR for differential diagnosis of benign and malignant breast masses. LGV, quantitative enhanced gray value of lesions; LBR, lesion to background grey value ratio; ROC, receiver operating characteristic; $\mathrm{AUC}$, area under the curve.
Between the early and second phases, $37.8 \%(31 / 82)$ of the malignant lesions exhibited the washout pattern, while $34.5 \%(10 / 29)$ of the benign lesions manifested as a persistent pattern. When the delayed phase was added, the washout pattern of malignant lesions and the persistent pattern of benign lesions decreased by $19.5 \%$ and $13.8 \%$, respectively.

Next, we explored the kinetic enhancement pattern of LBRs in consecutive phases (Figure 8). Between the early and second phases, malignant lesions commonly showed a washout enhancement pattern $(45 / 82,54.9 \%)$, whereas benign lesions mainly showed persistent enhancement patterns $(17 / 29,58.6 \%)$. Comparing the kinetic enhancement patterns in the early and second phase versus the second and delayed phase showed that the percentage of washout and persistent patterns decreased slightly in malignancies by $9.8 \%$ and in benign lesions by $7.9 \%$.

On combining LGV and LBR with kinetic enhancement patterns, the AUC showed improvement compared to an analysis based on LGV and LBR alone (AUC >0.7; Table 5), and LGV combined with kinetic enhancement achieved a higher specificity than LGV alone. LGV combined with the kinetic enhancement ${ }_{\mathrm{T} 1-\mathrm{T} 2}$ had a higher sensitivity, NPV, 
Table 4 Comparing the diagnostic performance of LGV and LBR combined among different imaging phases

\begin{tabular}{|c|c|c|c|c|c|c|}
\hline Imaging & AUC & Sensitivity (\%) & Specificity (\%) & PPV (\%) & NPV (\%) & Accuracy (\%) \\
\hline $\mathrm{LGV}_{\mathrm{T} 1}+\mathrm{LGV}_{\mathrm{T} 2}+\mathrm{LGV}_{\mathrm{T} 3}$ & 0.687 & 86.5 & 51.7 & 83.5 & 57.6 & 77.4 \\
\hline $\mathrm{LBR}_{\mathrm{T} 1}+\mathrm{LBR}_{\mathrm{T} 2}$ & 0.692 & 74.3 & 58.6 & 83.5 & 44.7 & 70.2 \\
\hline $\mathrm{LBR}_{\mathrm{T} 1}+\mathrm{LBR}_{\mathrm{T} 2}+\mathrm{LBR}_{\mathrm{T} 3}$ & 0.683 & 93.9 & 37.9 & 81.0 & 68.7 & 79.2 \\
\hline
\end{tabular}

$\mathrm{LGV}_{\mathrm{T} 1}, \mathrm{LGV}_{\mathrm{T} 2}$, LGV $\mathrm{T}_{3}$ : the LGV at the early, second, and delayed phase, respectively; $\mathrm{LBR}_{\mathrm{T} 1}, \mathrm{LBR}_{\mathrm{T} 2}$, $\mathrm{LBR}_{\mathrm{T} 3}$ : the LBR at the early, second, and delayed phase, respectively. LGV, quantitative enhanced gray value of lesions; LBR, lesion to background grey value ratio; AUC, area under the curve; PPV, positive-predictive value; NPV, negative-predictive value.

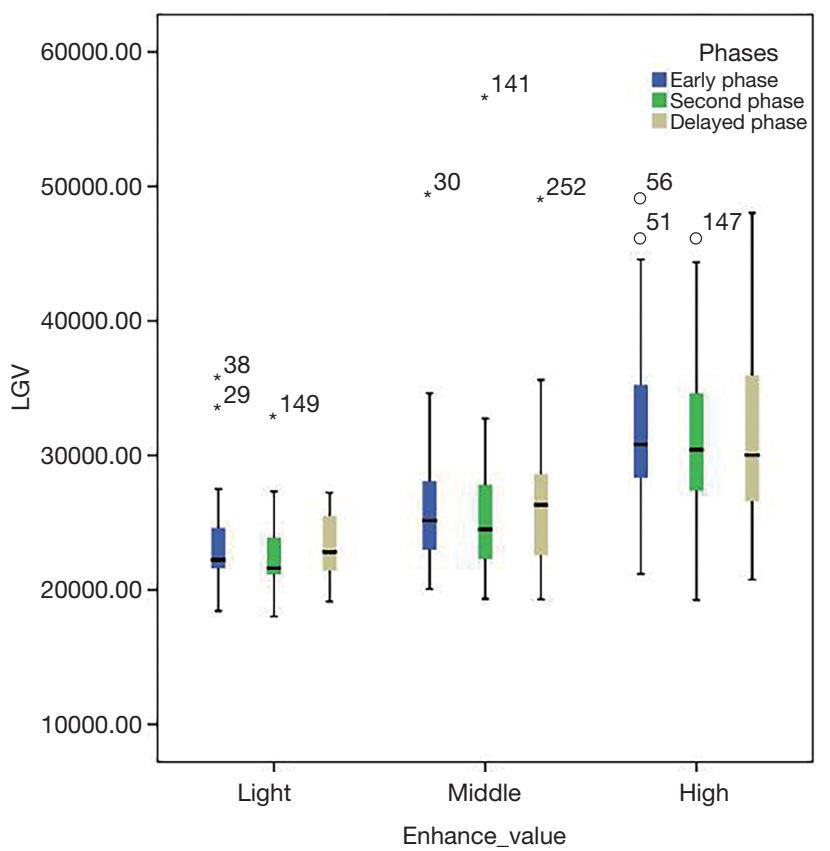

Figure 5 Comparison of the LGV enhancement value parameters with the qualitative evaluation by the reader. The asterisks represented the extremes more than three box lengths from the end of the box. The circles denoted the outliers between one and a half and three box lengths from the end of the box. LGV, quantitative enhanced gray value of lesions.

and accuracy $(84.1 \%, 59.3 \%$, and $79.2 \%$, respectively) than those of the three phases. The LGV combined with kinetic enhancement $_{\mathrm{T} 1-\mathrm{T} 3}$ achieved a higher specificity and PPV (75.8\% and $88.7 \%$, respectively) (Table 5 ).

LBR combined with kinetic enhancement $\mathrm{T} 1-\mathrm{T} 2_{2}$ had the lowest sensitivity and accuracy $(63.4 \%$ and $66.0 \%)$ compared to other combinations. However, LBR combined with kinetic enhancement $\mathrm{T}_{\mathrm{T} 1 \mathrm{~T} 3}$ achieved the highest sensitivity and accuracy (90.2\% and $82.0 \%$ ) (Table 5).

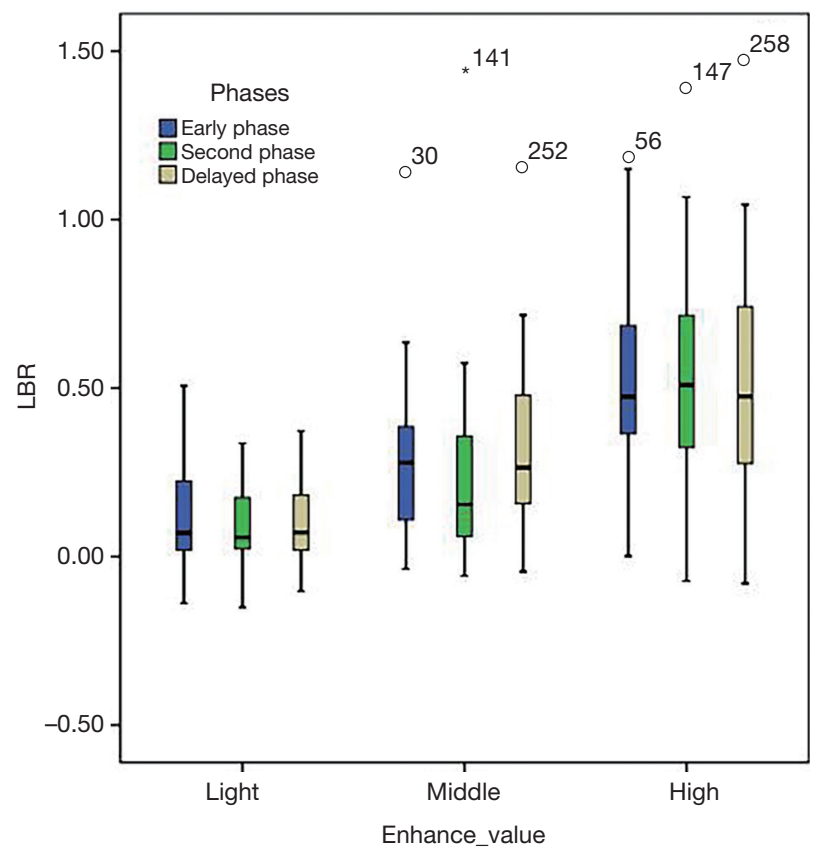

Figure 6 Comparison of the LBR enhancement value parameters with the qualitative evaluation by the reader. The asterisks represented the extremes more than three box lengths from the end of the box. The circles denoted the outliers between one and a half and three box lengths from the end of the box. LBR, lesion to background grey value ratio.

\section{Discussion}

This study showed that the mean LGV of malignant lesions was higher than that of benign lesions, consistent with previous studies $(10,11)$. Tsutsui et al. reported that a high micro-vessel density was frequently found in breast cancers with vascular endothelial VEGF expression (12), which would explain the higher enhancement of signal we observed. Remarkably, the mean LGV of malignant lesions 

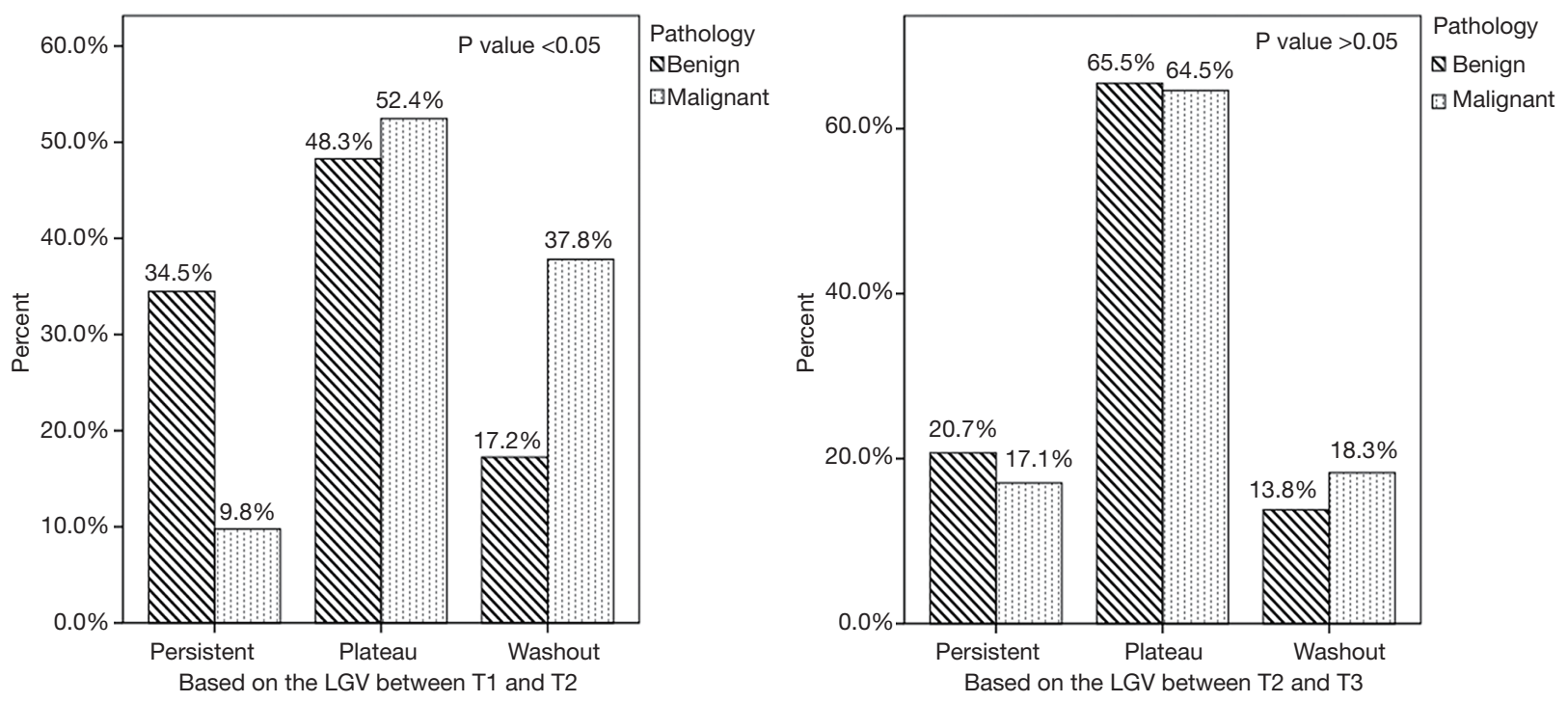

Figure 7 Enhancement patterns of two consecutive imaging phases based on the LGV. LGV, quantitative enhanced gray value of lesions.
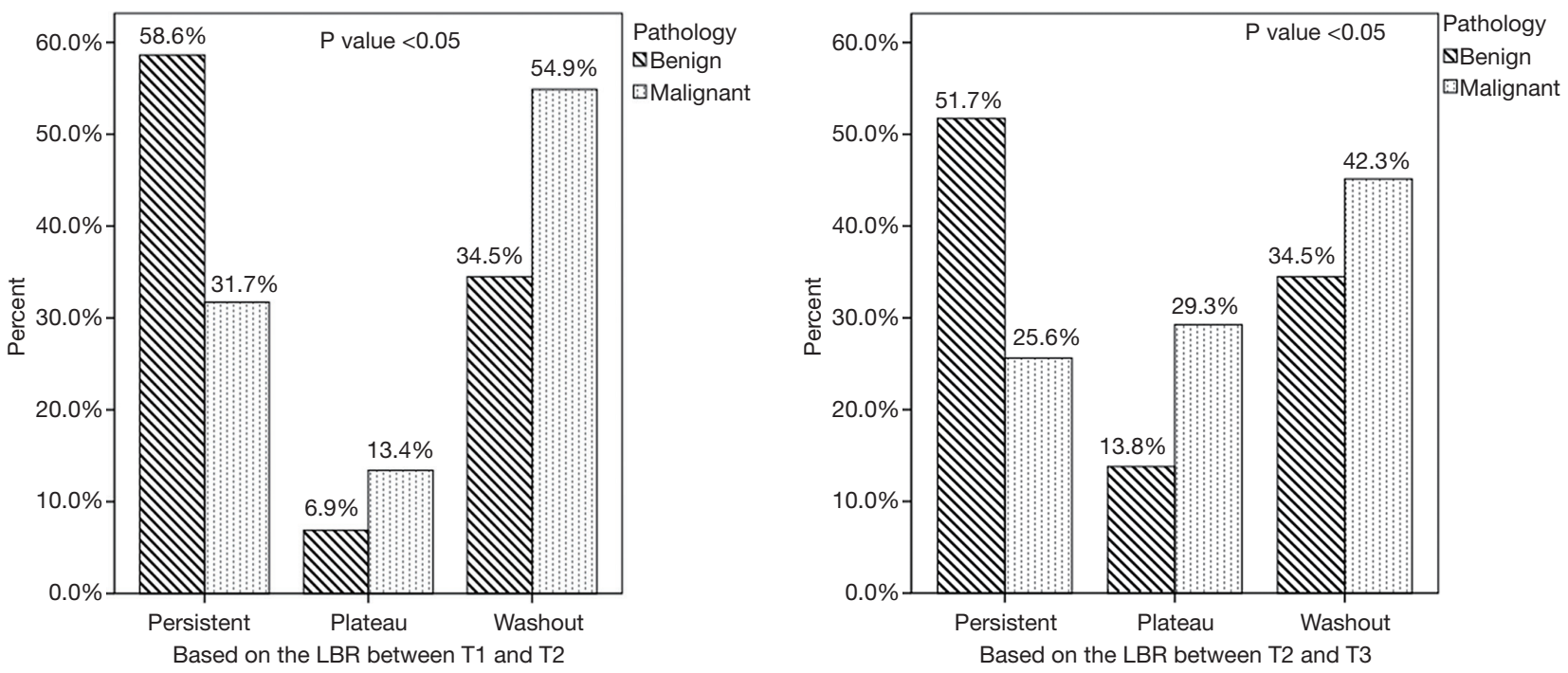

Figure 8 Enhancement patterns of two consecutive imaging phases based on the LBR. LBR, lesion to background grey value ratio.

reached its peak in the early phase and decreased over time, which might be because of the many new vessel networks in malignant lesions reabsorbing/consuming contrast agent rapidly, resulting in a significantly enhanced lesion in the early phase (16). On the contrary, the mean LGV of benign lesions was lowest in the early phase and increased over time.

The LGV diagnostic performance was much better in the early phase than in the other two phases, especially in accuracy and sensitivity. Based on LGV alone, the AUC, sensitivity, and accuracy of CEM reached their peak in the early phase, and the specificity was lowest in the delayed phase. Further, the diagnostic performance of LGV in the delayed phase was consistent with that in the second phase. Our findings were similar to several studies, which found the first post-contrast acquisition was sufficient to distinguish between malignant and benign breast lesions $(13,17,18)$.

The degree of enhancement depends on image acquisition time. LGV was combined with the change at different scan phases, and it was found that the diagnostic 
Table 5 The diagnostic performance of lesion LGV and LBR combined with enhancement and kinetic enhancement patterns

\begin{tabular}{|c|c|c|c|c|c|c|}
\hline Imaging & AUC & Sensitivity (\%) & Specificity (\%) & PPV (\%) & NPV (\%) & Accuracy (\%) \\
\hline $\mathrm{LGV}_{\mathrm{T} 1}+\mathrm{LGV}_{\mathrm{T} 2}+\mathrm{LGV}_{\mathrm{T} 3}+$ enhancement $_{\mathrm{T} 1-\mathrm{T} 3}$ & 0.771 & 67.0 & 75.8 & 88.7 & 44.8 & 69.3 \\
\hline $\mathrm{LBR}_{\mathrm{T} 1}+\mathrm{LBR}_{\mathrm{T} 2}+$ enhancement $_{\mathrm{T} 1-\mathrm{T} 2}$ & 0.749 & 63.4 & 75.0 & 89.6 & 37.5 & 66.0 \\
\hline $\mathrm{LBR}_{\mathrm{T} 1}+\mathrm{LBR}_{\mathrm{T} 2}+\mathrm{LBR}_{\mathrm{T} 3}+$ enhancement $_{\mathrm{T} 1-\mathrm{T} 3}$ & 0.783 & 90.2 & 54.1 & 87.0 & 61.9 & 82.0 \\
\hline
\end{tabular}

$\mathrm{LGV}_{\mathrm{T} 1}, \mathrm{LGV}_{\mathrm{T} 2}, \mathrm{LGV}_{\mathrm{T} 3}$ : the LGV at the early, second, and delayed phase, respectively; $\mathrm{LBR}_{\mathrm{T} 1}, \mathrm{LBR}_{\mathrm{T} 2}$, $\mathrm{LBR}_{\mathrm{T} 3}$ : the LBR at the early, second, and delayed phase, respectively; enhancement ${ }_{\mathrm{T} 1-\mathrm{T} 2}$ : the kinetic enhancement patterns between the early and second phase based on the LGV and LBR, respectively; enhancement ${ }_{\mathrm{T1-T3}}$ : the kinetic enhancement patterns among the early, second, and delayed phase based on the LGV and LBR, respectively. LGV, quantitative enhanced gray value of lesions; LBR, lesion to background grey value ratio; AUC, area under the curve; PPV, positive-predictive value; NPV, negative-predictive value.

performance of $\mathrm{LGV}_{\mathrm{T} 1}+\mathrm{LGV}_{\mathrm{T} 2}$ was similar to that of LGV in the early phase alone. Lv et al. (11) quantitatively measured the gray values within the lesion's ROI for two phases, and using the grey value within the ROI for distinguishing benign and malignant breast lesions; they achieved a higher AUC and specificity (AUC: $0.795 v s$. 0.695; specificity: $79.8 \%$ vs. $44.8 \%$ ) and lower sensitivity $(66.3 \%$ vs. $95.1 \%)$ than that of $\mathrm{LGV}_{\mathrm{T} 1}+\mathrm{LGV}_{\mathrm{T} 2}$. The possible reason for this discrepancy was that our study only included breast masses, and the method of quantitative measurement was different. When the delayed phase was added, the sensitivity of $\mathrm{LGV}_{\mathrm{T} 1}+\mathrm{LGV}_{\mathrm{T} 2}+\mathrm{LGV}_{\mathrm{T} 3}$ was lower than that of $\mathrm{LGV}_{\mathrm{T} 1}+\mathrm{LGV}_{\mathrm{T} 2}$ and higher than the previous study of $\mathrm{Lv}$ et al. (86.5\% vs. $95.1 \%$ vs. $69.5 \%$ ) (11), while the AUC, specificity, and PPV remained similar. We suggest distinguishing benign and malignant breast masses based on LGV alone, as the delayed phase does not improve diagnostic specificity.

Since we could not separate the exact pixel values of normal glandular tissue superimposed over enhanced lesions, the enhanced grey values were based on the bounded lesions. Therefore, we further explored the effect of BPEs at different phases and found that the difference in BPEs was not significantly different in malignant and benign lesions in each phase $(\mathrm{P}>0.05)$. The mean LBR of malignant lesions reached its peak in the early phase and decreased over time, while the mean LBR of benign lesions was lowest in the early phase and increased after that. Like LGV, the diagnostic performance of LBR was much better in the early phase than in the other two phases. Rudnicki et al. (1) found that the percentage signal difference between the enhancing lesion and background (\%RS) was likely to distinguish invasive malignant lesions from benign and intraductal lesions, which is similar to our finding.
Compared to Lv et al. (11), our study achieved a higher PPV based on LBR and a higher sensitivity based on $\mathrm{LBR}_{\mathrm{T} 1}$ and $\mathrm{LBR}_{\mathrm{T} 1}+\mathrm{LBR}_{\mathrm{T} 2}+\mathrm{LBR}_{\mathrm{T} 3}$. However, the AUC, specificity, and NPV of LBR alone and LBR with kinetic enhancement were lower than that reported by Lv et al. (11).

Analysis based on LBR alone revealed that while the AUC, sensitivity, NPV, and accuracy decreased over time, the specificity and PPV remained almost the same, indicating the early phase was still the best phase for breast masses. When the delayed phase was added, the sensitivity and PPV of $\mathrm{LBR}_{\mathrm{T} 1}+\mathrm{LBR}_{\mathrm{T} 2}+\mathrm{LBR}_{\mathrm{T} 3}$ increased by $19.6 \%$ and $24.0 \%$, respectively, while the specificity decreased by $20.7 \%$. The AUC, NPV, and accuracy did not differ between the $\mathrm{LBR}_{\mathrm{T} 1}+\mathrm{LBR}_{\mathrm{T} 2}$ and $\mathrm{LBR}_{\mathrm{T} 1}+\mathrm{LBR}_{\mathrm{T} 2}+\mathrm{LBR}_{\mathrm{T} 3}$, and the diagnostic performance of all combinations of LBR was similar to that of the LGV combinations.

Using the time-signal intensity curves (TIC) of MRI as a model, we can understand better the kinetic enhancement patterns of CEM and comprehend its applications. TIC is useful for distinguishing between benign and malignant lesions in breast MRI studies (19-21). While malignant lesions commonly demonstrate a higher percentage of washout enhancement patterns in TIC, a persistent enhancement pattern is significantly more likely to indicate a benign lesion $(19,22,23)$. In our study, based on the LGV, the most common enhancement patterns were consistent for both malignant and benign lesions between the early and second phases (52.4\% vs. $48.3 \%$ ) and the second and delayed phases (64.6\% vs. 65.4\%). However, our results are different from previous MRI studies because bilateral breasts were not scanned simultaneously, and we obtained images in three phases.

In terms of the kinetic enhancement of CEM, our results differ from those of Deng et al. (10), which may be 
attributed to the fact that we only enrolled female patients with breast masses. They found that when the CESM enhancement pattern was evaluated at two relative points between the early phase ( $2 \mathrm{~min}$ after the injection of the contrast medium on CC views) and the delayed phase (4 min after contrast medium injection on MLO views), the relative enhancement patterns of benign and malignant lesions were $15.91 \%$ vs. $17.92 \%$ in the elevated pattern, $2.27 \%$ vs. $12.26 \%$ in the steady pattern, $9.09 \%$ vs. $62.26 \%$ in the depressed pattern. Between the early and second phases, malignant and benign lesions in our study exhibited a lower proportion of the washout pattern $(37.8 \%)$ and persistent pattern $(34.5 \%)$, and when the delayed phase was added, the proportion of washout patterns $(18.3 \%)$ and persistent patterns $(20.7 \%)$ decreased. In terms of LBR kinetic enhancement (T1-T2; T2-T3), benign lesions manifested more persistent patterns between $\mathrm{T} 1-\mathrm{T} 2$ than that between T2-T3 (58.6\% vs. 51.7\%) and the proportion of washout patterns in malignant lesions between T1-T2 also exceeded that between T2-T3 (54.9\% vs. $42.3 \%)$. It is difficult to distinguish benign from malignant lesions based on kinetic enhancement by LGV or LBR. Moreover, a delayed phase cannot improve the probability of typical kinetic enhancement patterns of benign and malignant masses with persistent and washout patterns.

We further explored whether the combination of LGV and kinetic enhancement patterns could increase breast masses' diagnostic efficiency. Compared with the $\mathrm{LGV}_{\mathrm{T} 1}+$ $\mathrm{LGV}_{\mathrm{T} 2}+$ kinetic enhancement $\mathrm{T} 1-\mathrm{T} 2_{2}$ and $\mathrm{LGV}$ alone, $\mathrm{LGV}_{\mathrm{T} 1}$ $+\mathrm{LGV}_{\mathrm{T} 2}+\mathrm{LGV}_{\mathrm{T} 3}+$ kinetic enhancement ${ }_{\mathrm{T} 1-\mathrm{T} 3}$ is likely to be the preferable combination with the highest specificity and greatest ability to separate the malignant masses from benign ones.

LBR combined with kinetic enhancement obtained higher specificity than $\mathrm{LBR}_{\mathrm{T} 1}+\mathrm{LBR}_{\mathrm{T} 2}$ and $\mathrm{LBR}_{\mathrm{T} 1}+\mathrm{LBR}_{\mathrm{T} 2}$ $+\mathrm{LBR}_{\mathrm{T} 3}$, and when the delayed phase was added, the $\mathrm{LBR}_{\mathrm{T} 1}$ $+\mathrm{LBR}_{\mathrm{T} 2}+\mathrm{LBR}_{\mathrm{T} 3}+$ kinetic enhancement ${ }_{\mathrm{T} 1-\mathrm{T} 3}$ showed higher sensitivity and accuracy and lower specificity than $\mathrm{LBR}_{\mathrm{T} 1}+$ $\mathrm{LBR}_{\mathrm{T} 2}+$ kinetic enhancement ${ }_{\mathrm{T} 1-\mathrm{T} 2}$. We hypothesized that in terms of the BPE effect among different phases, the addition of the delayed phase severely reduces the specificity of LBR despite the increase in sensitivity, thus does not improve the ability to distinguish benign and malignant lesions.

AGD is related to cancer incidence. Although a second MLO view was added, the mean AGD was $1.92 \pm 0.05$ mGy per view below the limits set by regulatory institutions (24) and was lower than that of previous studies $(25,26)$.
However, with a second MLO view in our study, the total AGD per patient increased. According to the Biological Effects of Ionizing Radiation VII committee (24), the CEM poses little additional risk for cancer incidence and mortality. However, the addition of a second MLO is not recommended, bearing in mind that it does not provide any additional help in reaching a diagnosis and will only increase the radiation dose.

Our study has several limitations. First, this study was an analysis of breast masses only. Second, the delayed phase scan increased the AGD of patients. Therefore, we only chose one delayed phase at 7 minutes instead of multiple phases, which may not fully reflect the delayed phase's optimal efficiency. Third, this study lacked a comparison with MRI; for example, comparing the kinetic enhancement of the CEM delayed phase and the TIC of MRI. Fourth, this study only included single masses due to an insufficiency of cases.

Moreover, there was no subdivision of the internal enhancement features of the masses. Whole lesions were included within the ROIs, which means that the necrotic areas of uneven or circular enhancement were not excluded. Fifth, due to the automatic exposure mode, the consistency of each breast measurement's time duration could not be guaranteed, and finally, we only used quantitative features of the masses, ignoring morphological features.

\section{Conclusions}

The diagnostic performance of LGV and LBR reached a peak at the early and second phases. The malignant and benign lesions had the highest characteristic kinetic enhancement rate between the early and second phases, such as washout patterns in malignancies and persistent patterns in benign lesions. In our study, the delayed phase's addition did not significantly improve the diagnostic performance of CEM, indicating that a routine scan with two phases is sufficient for clinical diagnosis. The quantitative analysis combined with kinetic enhancement between the early and second phases has great potential for distinguishing malignant lesions from benign ones.

\section{Acknowledgments}

Funding: This work was supported by Clinical Research Start-up Program of Southern Medical University sponsored by High-Level University Construction Funding of Guangdong Provincial Department of Education 
(LC2016ZD018), Clinical Research Start-Up Program of Southern Medical University Nanfang Hospital (2020CR009), Natural Science Funding of Guangdong Province (2018A0303130215), Natural Science Funding of Guangdong Province (2019A1515011168) and National Key Research and Development Program of China (2019YFC0121903).

\section{Footnote}

Conflicts of Interest: All authors have completed the ICMJE uniform disclosure form (available at http://dx.doi. org/10.21037/qims-20-1092). The authors have no conflicts of interest to declare.

Ethical Statement: The authors are accountable for all aspects of the work in ensuring that questions related to the accuracy or integrity of any part of the work are appropriately investigated and resolved. The study was conducted following the Declaration of Helsinki (as revised in 2013). The Ethics Committee of Nanfang Hospital, Southern Medical University approved this prospective study (NFEC-2017-136), and written informed consent was received from all enrolled patients.

Open Access Statement: This is an Open Access article distributed in accordance with the Creative Commons Attribution-NonCommercial-NoDerivs 4.0 International License (CC BY-NC-ND 4.0), which permits the noncommercial replication and distribution of the article with the strict proviso that no changes or edits are made and the original work is properly cited (including links to both the formal publication through the relevant DOI and the license). See: https://creativecommons.org/licenses/by-nc-nd/4.0/.

\section{References}

1. Rudnicki W, Heinze S, Niemiec J, Kojs Z, Sas-Korczynska B, Hendrick E, Luczynska E. Correlation between quantitative assessment of contrast enhancement in contrast-enhanced spectral mammography (CESM) and histopathology-preliminary results. Eur Radiol 2019;29:6220-6.

2. Li L, Roth R, Germaine P, Ren S, Lee M, Hunter K, Tinney E, Liao L. Contrast-enhanced spectral mammography (CESM) versus breast magnetic resonance imaging (MRI): a retrospective comparison in 66 breast lesions. Diagn Interv Imaging 2017;98:113-23.
3. Mohamed Kamal R, Hussien Helal M, Wessam R, Mahmoud Mansour S, Godda I, Alieldin N. Contrastenhanced spectral mammography: impact of the qualitative morphology descriptors on the diagnosis of breast lesions. Eur J Radiol 2015;84:1049-55.

4. Fallenberg EM, Schmitzberger FF, Amer H, IngoldHeppner B, Balleyguier C, Diekmann F, Engelken F, Mann RM, Renz DM, Bick U, Hamm B, Dromain C. Contrastenhanced spectral mammography vs. mammography and MRI - clinical performance in a multi-reader evaluation. Eur Radiol 2017;27:2752-64.

5. Travieso-Aja MM, Maldonado-Saluzzi D, NaranjoSantana P, Fernández-Ruiz C, Severino-Rondón W, Rodríguez Rodríguez M, Luzardo OP. Evaluation of the applicability of BI-RADS® MRI for the interpretation of contrast-enhanced digital mammography. Radiologia 2019;61:477-88.

6. Baltzer PA, Benndorf M, Dietzel M, Gajda M, Camara O, Kaiser WA. Sensitivity and specificity of unenhanced MR mammography (DWI combined with T2-weighted TSE imaging, ueMRM) for the differentiation of mass lesions. Eur Radiol 2010;20:1101-10.

7. Kamal RM, Helal MH, Mansour SM, Haggag MA, Nada OM, Farahat IG, Alieldin NH. Can we apply the MRI BI-RADS lexicon morphology descriptors on contrast-enhanced spectral mammography? Br J Radiol 2016;89:20160157.

8. Cozzi A, Schiaffino S, Giorgi Rossi P, Sardanelli F. Breast cancer screening: in the era of personalized medicine, age is just a number. Quant Imaging Med Surg 2020;10:2401-7.

9. Sorin V, Yagil Y, Shalmon A, Gotlieb M, Faermann R, Halshtok-Neiman O, Sklair-Levy M. Background parenchymal enhancement at contrast-enhanced spectral mammography (CESM) as a breast cancer risk factor. Acad Radiol 2020;27:1234-40.

10. Deng CY, Juan YH, Cheung YC, Lin YC, Lo YF, Lin G, Chen SC, Ng SH. Quantitative analysis of enhanced malignant and benign lesions on contrast-enhanced spectral mammography. Br J Radiol 2018;91:20170605.

11. Lv Y, Chi X, Sun B, Lin S, Xing D. Diagnostic value of quantitative gray-scale analysis of contrast-enhanced spectral mammography for benign and malignant breast lesions. J Comput Assist Tomogr 2020;44:405-12.

12. Tsutsui S, Inoue H, Yasuda K, Suzuki K, Takeuchi H, Nishizaki T, Higashi H, Era S, Mori M. Angiopoietin 2 expression in invasive ductal carcinoma of the breast: its relationship to the VEGF expression and microvessel density. Breast Cancer Res Treat 2006;98:261-6. 
13. Jong RA, Yaffe MJ, Skarpathiotakis M, Shumak RS, Danjoux NM, Gunesekara A, Plewes DB. Contrastenhanced digital mammography: initial clinical experience. Radiology 2003;228:842-50.

14. Huang JS, Pan HB, Yang TL, Hung BH, Chiang CL, Tsai MY, Chou CP. Kinetic patterns of benign and malignant breast lesions on contrast enhanced digital mammogram. PLoS One 2020;15:e0239271.

15. Morris EA, Comstock CE, Lee CH, et al. ACR BI-RADS ${ }^{\circledR}$ Magnetic Resonance Imaging. In: ACR BI-RADS ${ }^{\circledR}$ Atlas, Breast Imaging Reporting and Data System. Reston: American College of Radiology, 2013. Available online: https://www.acr.org/Clinical-Resources/Reporting-andData-Systems/Bi-Rads/Permissions

16. Du J, Li FH, Fang H, Xia JG, Zhu CX. Correlation of real-time gray scale contrast-enhanced ultrasonography with microvessel density and vascular endothelial growth factor expression for assessment of angiogenesis in breast lesions. J Ultrasound Med 2008;27:821-31.

17. Buckley DL, Mussurakis S, Horsman A. Effect of temporal resolution on the diagnostic efficacy of contrast-enhanced MRI in the conservatively treated breast. J Comput Assist Tomogr 1998;22:47-51.

18. Sardanelli F, Rescinito G, Giordano GD, Calabrese M, Parodi RC. MR dynamic enhancement of breast lesions: high temporal resolution during the first-minute versus eight-minute study. J Comput Assist Tomogr 2000;24:724-31.

19. Kuhl CK, Mielcareck P, Klaschik S, Leutner C, Wardelmann E, Gieseke J, Schild HH. Dynamic breast MR imaging: are signal intensity time course data useful for differential diagnosis of enhancing lesions? Radiology 1999;211:101-10.

Cite this article as: $\mathrm{Xu} W$, Zheng B, Chen W, Wen C, Zeng H, He Z, Qin G, Li Y. Can the delayed phase of quantitative contrast-enhanced mammography improve the diagnostic performance on breast masses? Quant Imaging Med Surg 2021;11(8):3684-3697. doi: 10.21037/qims-20-1092
20. Yabuuchi H, Matsuo Y, Okafuji T, Kamitani T, Soeda H, Setoguchi T, Sakai S, Hatakenaka M, Kubo M, Sadanaga $\mathrm{N}$, Yamamoto H, Honda H. Enhanced mass on contrastenhanced breast MR imaging: Lesion characterization using combination of dynamic contrast-enhanced and diffusion-weighted MR images. J Magn Reson Imaging 2008;28:1157-65.

21. Abe H, Mori N, Tsuchiya K, Schacht DV, Pineda FD, Jiang Y, Karczmar GS. Kinetic analysis of benign and malignant breast lesions with ultrafast dynamic contrastenhanced MRI: comparison with standard kinetic assessment. AJR Am J Roentgenol 2016;207:1159-66.

22. Shimauchi A, Abe H, Schacht DV, Yulei J, Pineda FD, Jansen SA, Ganesh R, Newstead GM. Evaluation of kinetic entropy of breast masses initially found on MRI using whole-lesion curve distribution data: comparison with the standard kinetic analysis. Eur Radiol 2015;25:2470-8.

23. Wang LC, DeMartini WB, Partridge SC, Peacock S, Lehman CD. MRI-detected suspicious breast lesions: predictive values of kinetic features measured by computeraided evaluation. AJR Am J Roentgenol 2009;193:826-31.

24. National Research Council. Health risks from exposure to low levels of ionizing radiation: BEIR VII phase 2. Washington, DC: The National Academies Press, 2006.

25. James JR, Pavlicek W, Hanson JA, Boltz TF, Patel BK. Breast radiation dose with CESM compared with 2D FFDM and 3D tomosynthesis mammography. AJR Am J Roentgenol 2017;208:362-72.

26. Phillips J, Mihai G, Hassonjee SE, Raj SD, Palmer MR, Brook A, Zhang D. Comparative dose of contrastenhanced spectral mammography (CESM), digital mammography, and digital breast tomosynthesis. AJR Am J Roentgenol 2018;211:839-46. 